\title{
Étude des différences de corps convexes plans
}

\author{
par Yves Martinez-Maure (Rueil-Malmaison)
}

\begin{abstract}
We characterize the linear space $\mathcal{H}$ of differences of support functions of convex bodies of $\mathbb{E}^{2}$ and we consider every $h \in \mathcal{H}$ as the support function of a generalized hedgehog (a rectifiable closed curve having exactly one oriented support line in each direction). The mixed area (for plane convex bodies identified with their support functions) has a symmetric bilinear extension to $\mathcal{H}$ which can be interpreted as a mixed area for generalized hedgehogs. We study generalized hedgehogs and we extend the Minkowski inequality.
\end{abstract}

Introduction. L'ensemble $\mathcal{C}$ des corps convexes du plan euclidien $\mathbb{E}^{2}$ ne constitue pas un espace vectoriel. Par contre, son symétrisé pour l'addition de Minkowski constitue un espace vectoriel $\mathcal{D}$ dans lequel $\mathcal{C}$ peut être envisagé comme un cône convexe et l'aire mixte comme une forme bilinéaire symétrique. Cette remarque suggère d'étendre la théorie de Brunn-Minkowski plane à $\mathcal{D}$ par l'introduction d'une notion géométrique adéquate. Ce problème fut partiellement résolu dès 1937 par H. Geppert qui a introduit à cet effet la notion de "domaine supportable" (stützbarer Bereich) [1]. Dans le même article, H. Geppert introduit la notion de "surface supportable" (stützbare Flächen) et donne des résultats analogues dans l'espace euclidien à 3 dimensions. Ce travail ne concerne par construction que des corps convexes dont les fonctions support vérifient certaines conditions de différentiabilité. Beaucoup plus tard et indépendamment, une approche analogue basée sur la notion de hérisson (enveloppe paramétrée par son application de Gauss) fut proposée dans l'espace euclidien à $n$ dimensions par R. Langevin, G. Levitt et H. Rosenberg [3]. Cette approche a été ensuite approfondie par l'auteur [4], mais toujours sous des conditions de différentiabilité restrictives.

Le principal objectif de cet article est de prouver que la différence formelle de deux corps convexes plans quelconques peut être considérée comme une

\footnotetext{
1991 Mathematics Subject Classification: 52A38, 53A04.

Key words and phrases: convex curve, support function, hedgehog, Brunn-Minkowski theory.
} 
courbe rectifiable admettant exactement une droite support orientée dans chaque direction et d'étendre les principaux résultats de la théorie de BrunnMinkowski plane à l'espace vectoriel formé par ces courbes.

La construction repose sur la notion de fonction support. Tout corps convexe $K$ de $\mathbb{E}^{2}$ s'identifie à sa fonction support $h_{K}: \mathbb{S}^{1} \rightarrow \mathbb{R}, u \mapsto$ $\sup \{\langle u, v\rangle \mid v \in K\}$. Cette fonction support est continue et la somme de Minkowski de deux corps convexes est définie par la somme de leurs fonctions support. Par conséquent, l'ensemble $\mathcal{C}$ des corps convexes de $\mathbb{E}^{2}$ s'identifie à un cône convexe $\mathcal{K}$ de $C\left(\mathbb{S}^{1}\right)$ et son symétrisé $\mathcal{D}$ pour l'addition de Minkowski au sous-espace $\mathcal{H}$ que $\mathcal{K}$ engendre dans $C\left(\mathbb{S}^{1}\right)$. Nous rappelons que :

(i) $C^{2}\left(\mathbb{S}^{1}\right) \subset \mathcal{H}$;

(ii) si une fonction $h \in C^{2}\left(\mathbb{S}^{1}\right)$ n'est pas toujours la fonction support d'une courbe convexe, elle peut toujours se voir comme celle de l'enveloppe de la famille de droites définie par $\langle x, u(\theta)\rangle=p(\theta)$, où $x=\left(x_{1}, x_{2}\right), u(\theta)=$ $(\cos \theta, \sin \theta)$ et $p(\theta)=h(u(\theta))$;

(iii) cette enveloppe $\mathcal{H}_{h}$, paramétrée par $x_{h}: \mathbb{S}^{1} \rightarrow \mathbb{E}^{2}, u(\theta) \mapsto x_{h}(\theta)$, où $x_{h}(\theta)=p(\theta) u(\theta)+p^{\prime}(\theta) u^{\prime}(\theta)$ est l'unique solution du système

$$
\left\{\begin{array}{l}
\langle x, u(\theta)\rangle=p(\theta) \\
\left\langle x, u^{\prime}(\theta)\right\rangle=p^{\prime}(\theta),
\end{array}\right.
$$

est appelée le hérisson de fonction support $h$;

(iv) son aire algébrique $a(h)$ est l'intégrale sur $\mathbb{E}^{2}-\mathcal{H}_{h}$ du nombre algébrique d'intersection $i_{h}(x)$ d'une demi-droite orientée d'origine $x$ avec $\mathcal{H}_{h}$ muni de son orientation transverse (nombre indépendant de la demidroite orientée pour un ouvert dense de directions) [3].

Dans la section 1, nous définissons des hérissons de $\mathbb{E}^{2}$ par une fonction quelconque de $\mathcal{H}$, c'est-à-dire par une différence de fonctions support de corps convexes plans. Nous parlons alors de hérissons généralisés. La notion d'aire s'étend aux hérissons généralisés de sorte que l'extension bilinéaire de l'aire mixte s'interprète encore comme une aire mixte. Nous étudions les hérissons généralisés et nous caractérisons les fonctions support de ceux qui sont convexes. Nous caractérisons également le sous-espace $\mathcal{H}$ de $C\left(\mathbb{S}^{1}\right)$. Enfin, dans la section 2 nous étudions l'extension aux hérissons généralisés de l'inégalité de Minkowski (et donc de l'inégalité isopérimétrique).

Je tiens à exprimer ici ma gratitude au rapporteur qui m'a fait découvrir le travail de H. Geppert.

1. Les hérissons plans généralisés. Étudions comment la frontière d'un corps convexe $K$ de $\mathbb{E}^{2}$ est déterminée par sa fonction support $h_{K}$. Nous savons que $h=h_{K}$ est la restriction à $\mathbb{S}^{1}$ d'une fonction sous-linéaire $\varphi: \mathbb{E}^{2} \rightarrow \mathbb{R}$ dont les sous-différentiels $\partial \varphi(u)=\left\{x \in \mathbb{E}^{2} \mid \forall v \in \mathbb{E}^{2}, \varphi(v) \geq\right.$ $\varphi(u)+\langle x, v-u\rangle\}$ aux points $u$ de $\mathbb{S}^{1}$ sont les ensembles support $S_{K}(u)=$ 
$\{x \in K \mid\langle x, u\rangle=h(u)\}$ (cf. [5], Th. 1.7.4). La frontière de $K$ est donc la réunion des sous-différentiels $\partial \varphi(u(\theta))$. Comme $\varphi$ est une fonction convexe, toutes les dérivées directionnelles $\varphi^{\prime}(u(\theta) ; v)$ existent et on a $\partial \varphi(u(\theta))=$ $\left\{x \in \mathbb{E}^{2} \mid \forall v \neq 0_{\mathbb{E}^{2}}, \varphi^{\prime}(u(\theta) ; v) \geq\langle x, v\rangle\right\}$ (ibid., p. 25). Or, la dérivée à gauche $p_{\mathrm{g}}^{\prime}(\theta)$ et la dérivée à droite $p_{\mathrm{d}}^{\prime}(\theta)$ de la fonction $p(\theta)=h(u(\theta))$ peuvent s'écrire respectivement $-\varphi^{\prime}\left(u(\theta) ;-u^{\prime}(\theta)\right)$ et $\varphi^{\prime}\left(u(\theta) ; u^{\prime}(\theta)\right)$, et on a $\varphi^{\prime}\left(u(\theta) ; \alpha u(\theta)+\beta u^{\prime}(\theta)\right)=\alpha p(\theta)+\varepsilon \beta \varphi^{\prime}\left(u(\theta) ; \varepsilon u^{\prime}(\theta)\right)$, où $\varepsilon=\operatorname{sgn}(\beta)$. Par suite, ces dérivées $p_{\mathrm{g}}^{\prime}(\theta)$ et $p_{\mathrm{d}}^{\prime}(\theta)$ existent et on obtient $\partial \varphi(u(\theta))=\{x \in$ $\left.\mathbb{E}^{2} \mid \exists t \in\left[p_{\mathrm{g}}^{\prime}(\theta), p_{\mathrm{d}}^{\prime}(\theta)\right], x=p(\theta) u(\theta)+t u^{\prime}(\theta)\right\}$. La frontière de $K$ est donc la réunion des segments $\sigma_{h}(\theta)=\left[x_{h}^{-}(\theta), x_{h}^{+}(\theta)\right](\theta \in J=[0,2 \pi[)$, où $x_{h}^{-}(\theta)=p(\theta) u(\theta)+p_{\mathrm{g}}^{\prime}(\theta) u^{\prime}(\theta)$ et $x_{h}^{+}(\theta)=p(\theta) u(\theta)+p_{\mathrm{d}}^{\prime}(\theta) u^{\prime}(\theta)$, et le segment $\sigma_{h}(\theta)$ est l'ensemble support de vecteur normal $u(\theta)$.

Nous rappelons que cette frontière $\operatorname{Fr}(K)$ est une courbe fermée simple et rectifiable. Commençons par prouver que, pour tout $h \in \mathcal{H}$, les segments $\sigma_{h}(\theta)$ sont bien définis et forment encore une courbe fermée rectifiable $\mathcal{H}_{h}$. La preuve repose sur le résultat suivant :

Proposition 1. Pour tout $h \in \mathcal{H}$, les propriétés suivantes sont satisfaites:

(1) la fonction $p(\theta)=h(u(\theta))$ est lipschitzienne sur $\mathbb{R}$;

(2) $p$ admet une dérivée à gauche $p_{\mathrm{g}}^{\prime}$ (resp. à droite $\left.p_{\mathrm{d}}^{\prime}\right)$ continue à gauche (resp. à droite) sur $\mathbb{R}$, et : $\forall \theta \in \mathbb{R}, p_{\mathrm{g}}^{\prime}(\theta)=\lim _{\alpha \rightarrow \theta^{-}} p_{\mathrm{d}}^{\prime}(\alpha)$ et $p_{\mathrm{d}}^{\prime}(\theta)=$ $\lim _{\alpha \rightarrow \theta^{+}} p_{\mathrm{g}}^{\prime}(\alpha)$

(3) la famille $\left(\left(p_{\mathrm{d}}^{\prime}-p_{\mathrm{g}}^{\prime}\right)(\theta)\right)_{\theta \in J=[0,2 \pi[\text { est absolument sommable; }}$

(4) les fonctions $p_{\mathrm{g}}^{\prime}$ et $p_{\mathrm{d}}^{\prime}$ sont à variation bornée sur $I=[0,2 \pi]$.

Preuve résumée. Il suffit d'établir ce résultat pour $h \in \mathcal{K}$. Reprenons la fonction $h=h_{K}$ et les notations introduites au début de cette section.

(1) La propriété (1) découle de la convexité de la fonction $\varphi$ (cf. [5], Th. 1.5.1).

(2) Comme la fonction $\varphi_{\theta}(t)=\varphi\left(u(\theta)+t u^{\prime}(\theta)\right)$ est convexe sur $\mathbb{R}$, elle admet sur $\mathbb{R}$ une dérivée à gauche $\left(\varphi_{\theta}\right)_{\mathrm{g}}^{\prime}$ continue à gauche et une dérivée à droite $\left(\varphi_{\theta}\right)_{\mathrm{d}}^{\prime}$ continue à droite, et ces dérivées vérifient $\left(\varphi_{\theta}\right)_{\mathrm{g}}^{\prime} \leq\left(\varphi_{\theta}\right)_{\mathrm{d}}^{\prime}$. La propriété (2) s'en déduit directement en exprimant ces dérivées à l'aide des fonctions $p, p_{\mathrm{g}}^{\prime}$ et $p_{\mathrm{d}}^{\prime}$.

(3) La propriété (3) résulte du fait que la courbe fermée simple $\operatorname{Fr}(K)$ est rectifiable et réunion des segments $\sigma_{h}(\theta)=\left[x_{h}^{-}(\theta), x_{h}^{+}(\theta)\right]$ qui ne peuvent se rencontrer qu'en leurs extrémités.

(4) Sachant que pour toute suite strictement croissante $\left(\theta_{i}\right)_{i \geq 0}$ de $I$, les segments $\sigma_{h}\left(\theta_{i}\right)$ sont disposés dans l'ordre croissant des indices sur la courbe $\operatorname{Fr}(K)$ orientée dans le sens direct, il en résulte aussi que les fonctions $x_{h}^{-}$ et $x_{h}^{+}$sont à variation bornée sur $I$. On en déduit que les fonctions $p_{\mathrm{g}}^{\prime} u^{\prime}$ et 
$p_{\mathrm{d}}^{\prime} u^{\prime}$ sont également à variation bornée sur $I$ en utilisant la propriété (1). En observant par ailleurs que les fonctions $p_{\mathrm{g}}^{\prime}$ et $p_{\mathrm{d}}^{\prime}$ sont nécessairement bornées, on en déduit enfin la propriété (4).

Prenons $h$ une fonction quelconque de $\mathcal{H}$. Les segments $\sigma_{h}(\theta)=$ $\left[x_{h}^{-}(\theta), x_{h}^{+}(\theta)\right]$ qui sont bien définis d'après la propriété (2), forment l'image de l'application

$$
x_{h}: D_{x_{h}} \rightarrow \mathbb{E}^{2}, \quad(\theta, t) \mapsto p(\theta) u(\theta)+t u^{\prime}(\theta),
$$

où $D_{x_{h}}=\left\{(\theta, t) \in J \times \mathbb{R} \mid\left(t-p_{\mathrm{g}}^{\prime}(\theta)\right)\left(t-p_{\mathrm{d}}^{\prime}(\theta)\right) \leq 0\right\} \cup\left\{\left(2 \pi, p_{\mathrm{g}}^{\prime}(2 \pi)\right)\right\}$. Notons que les points $A=\left(0, p_{\mathrm{g}}^{\prime}(0)\right)$ et $B=\left(2 \pi, p_{\mathrm{g}}^{\prime}(2 \pi)\right)$ ont la même image par $x_{h}$.

Montrons comment $x_{h}\left(D_{x_{h}}\right)$ peut se voir comme une courbe fermée de $\mathbb{E}^{2}$. Munissons pour cela l'ensemble $D_{x_{h}}$ de la distance $d$, définie par

$$
\begin{aligned}
& d\left(\left(\theta_{1}, t_{1}\right),\left(\theta_{2}, t_{2}\right)\right) \\
& \quad= \begin{cases}\left|t_{1}-t_{2}\right| & \text { si } \theta_{1}=\theta_{2}, \\
\theta_{j}-\theta_{i}+\left|p_{\mathrm{d}}^{\prime}\left(\theta_{i}\right)-t_{i}\right|+s\left(\theta_{i}, \theta_{j}\right)+\left|t_{j}-p_{\mathrm{g}}^{\prime}\left(\theta_{j}\right)\right| & \text { si } \theta_{i}<\theta_{j},\end{cases}
\end{aligned}
$$

où $s\left(\theta_{i}, \theta_{j}\right)=\sum_{\theta_{i}<\alpha<\theta_{j}}\left|\left(p_{\mathrm{d}}^{\prime}-p_{\mathrm{g}}^{\prime}\right)(\alpha)\right|$ (cf. propriété $\left.(3)\right)$, et observons que cette distance $d$ est telle que :

(i) l'application $d_{h}: D_{x_{h}} \rightarrow \mathbb{R}, M \mapsto d(A, M)$ est une isométrie de $D_{x_{h}}$ sur $I_{h}=\left[0, F_{h}\right]$, où $F_{h}=d_{h}(B)=2 \pi+\sum_{\theta \in J}\left|\left(p_{\mathrm{g}}^{\prime}-p_{\mathrm{d}}^{\prime}\right)(\theta)\right|$ (la vérification de ce point ne présente aucune difficulté particulière);

(ii) l'application $x_{h}$ est continue sur $D_{x_{h}}$ (se ramener à la continuité de l'application $D_{x_{h}} \rightarrow \mathbb{R},(\theta, t) \mapsto t$, puis utiliser la propriété (2)).

Nous pouvons ainsi définir l'application

$\gamma_{h}=x_{h} \circ d_{h}^{-1}: I_{h} \rightarrow \mathbb{E}^{2}, \quad \lambda \mapsto x_{h}(\theta(\lambda), t(\lambda)), \quad$ où $\quad(\theta(\lambda), t(\lambda))=d_{h}^{-1}(\lambda)$,

et affirmer qu'elle est continue sur $I_{h}$ et de même image que $x_{h}$. La courbe de $\mathbb{E}^{2}$ qu'elle définit est bien fermée puisque $x_{h}(A)=x_{h}(B)$, et nous pouvons donc énoncer le résultat suivant :

ThÉORÈme 1. Pour tout $h \in \mathcal{H}$, l'application $\gamma_{h}: I_{h} \rightarrow \mathbb{E}^{2}$ définit une courbe fermée $\mathcal{H}_{h}$ de $\mathbb{E}^{2}$, dont la réalisation géométrique $\gamma_{h}\left(I_{h}\right)$ est la réunion des segments $\sigma_{h}(\theta)=\left[x_{h}^{-}(\theta), x_{h}^{+}(\theta)\right] \quad(\theta \in J=[0,2 \pi[)$.

DÉfinition. Pour tout $h \in \mathcal{H}$, nous appellerons hérisson généralisé de fonction support $h$ la courbe fermée $\mathcal{H}_{h}$ de $\mathbb{E}^{2}$ dont chaque partie régulière est munie de l'orientation transverse pour laquelle le vecteur normal en un point $\gamma_{h}(\lambda)=x_{h}(\theta(\lambda), t(\lambda))$ n'est autre que $u(\theta(\lambda))$, et ensemble support de $\mathcal{H}_{h}$ dans la direction $u(\theta)$ le segment $\sigma_{h}(\theta)=\left[x_{h}^{-}(\theta), x_{h}^{+}(\theta)\right]$. 
ThÉORÈme 2. Pour tout $h \in \mathcal{H}$, le hérisson généralisé $\mathcal{H}_{h}$ est une courbe rectifiable, de longueur

$$
L(h)=\int_{0}^{F_{h}}\left\|\gamma_{h}^{\prime}(\lambda)\right\| d \lambda .
$$

Preuve résumée. Il suffit de prouver que l'application $\gamma_{h}$ est lipschitzienne (et donc absolument continue) sur $I_{h}$. Notons d'abord que les fonctions réelles $\theta$ et $t$, définies par la relation $(\theta(\lambda), t(\lambda))=d_{h}^{-1}(\lambda)$, sont 1-lipschitziennes sur $I_{h}$. En effet, pour $\theta(\lambda)<\theta(\mu)$, on a $|\mu-\lambda|=\mid \theta(\mu)-$ $\theta(\lambda) \mid+f(\lambda, \mu)$, où $f(\lambda, \mu)=\left|p_{\mathrm{d}}^{\prime}(\theta(\lambda))-t(\lambda)\right|+s(\theta(\lambda), \theta(\mu))+\mid t(\mu)-$ $p_{\mathrm{g}}^{\prime}(\theta(\mu)) \mid$, et on prouve à l'aide de la propriété (2) que $|t(\mu)-t(\lambda)| \leq$ $f(\lambda, \mu)$, en notant que le segment $[t(\lambda), t(\mu)]$ est inclus dans la réunion des segments $\left[t(\lambda), p_{\mathrm{d}}^{\prime}(\theta(\lambda))\right],\left[p_{\mathrm{g}}^{\prime}(\theta(\mu)), t(\mu)\right]$ et des segments $\left[p_{\mathrm{g}}^{\prime}(\theta), p_{\mathrm{d}}^{\prime}(\theta)\right]$, où $\theta \in] \theta(\lambda), \theta(\mu)[)$. On en déduit que l'application $\gamma_{h}$ est lipschitzienne sur $I_{h}$ en utilisant la propriété (1) et le fait que la fonction $t$ est bornée.

Lemme et notation. Soit $\mathcal{F}$ le sous-espace de $C\left(\mathbb{S}^{1}\right)$ défini par

$$
\mathcal{F}=\left\{h \in C\left(\mathbb{S}^{1}\right) \mid p(\theta)=h(u(\theta)) \text { vérifie les propriétés }(1)-(4)\right\} .
$$

Pour tout $h \in \mathcal{F}$, les fonctions $p_{\mathrm{g}}^{\prime}$ et $p_{\mathrm{d}}^{\prime}$ admettent en presque tout $\theta \in \mathbb{R}$ une dérivée commune que nous noterons $p^{\prime \prime}(\theta)$.

Preuve. Il résulte de la propriété (4) que les fonctions $p_{\mathrm{g}}^{\prime}$ et $p_{\mathrm{d}}^{\prime}$ sont presque partout dérivables. De plus, comme la fonction $p$ est également presque partout dérivable d'après la propriété $(1)$, les fonctions $p_{\mathrm{g}}^{\prime}$ et $p_{\mathrm{d}}^{\prime}$ coïncident presque partout, de sorte que leurs dérivées coïncident également presque partout.

ThÉORÈme 3 . Pour tout $h \in \mathcal{H}$, la longueur $L(h)$ du hérisson généralisé $\mathcal{H}_{h}$ est donnée par

$$
L(h)=\int_{0}^{2 \pi}\left|\left(p+p^{\prime \prime}\right)(\theta)\right| d \theta+\sum_{\theta \in J}\left|\left(p_{\mathrm{d}}^{\prime}-p_{\mathrm{g}}^{\prime}\right)(\theta)\right| .
$$

Preuve. Sur l'ensemble $T_{h}=\left\{\lambda \in I_{h} \mid t(\lambda) \neq p_{\mathrm{g}}^{\prime}(\theta(\lambda)), p_{\mathrm{d}}^{\prime}(\theta(\lambda))\right\}$, nous avons $\gamma_{h}^{\prime}=\operatorname{sgn}\left(\left(p_{\mathrm{d}}^{\prime}-p_{\mathrm{g}}^{\prime}\right) \circ \theta\right)\left(u^{\prime} \circ \theta\right)$, de sorte que

$$
\int_{T_{h}}\left\|\gamma_{h}^{\prime}(\lambda)\right\| d \lambda=\sum_{\theta \in J}\left|\left(p_{\mathrm{g}}^{\prime}-p_{\mathrm{d}}^{\prime}\right)(\theta)\right|
$$

Or, l'ensemble $D_{h}=\left\{\lambda \in I_{h} \mid p^{\prime}(\theta(\lambda))\right.$ existe $\}$ est disjoint de $T_{h}$ et la propriété (3) nous assure que $I_{h}-\left(D_{h} \cup T_{h}\right)$ est dénombrable, et donc de mesure nulle. Donc, il suffit d'établir que $\int_{D_{h}}\left\|\gamma_{h}^{\prime}(\lambda)\right\| d \lambda=\int_{0}^{2 \pi}\left|\left(p+p^{\prime \prime}\right)(\theta)\right| d \theta$. Or, $\theta^{\prime}(\lambda)=1$ pour presque tout $\lambda \in D_{h}$ et $\theta(\lambda)$ définit un homéomorphisme conservant la mesure de $D_{h}$ sur $\Delta_{h}=\left\{\theta \in I \mid p^{\prime}(\theta)\right.$ existe $\}$ (sachant que 
$\theta$ est 1-lipschitzienne et croissante sur $I_{h}$, on prouve la relation $\int_{\theta(X)} d \theta=$ $\int_{X} \theta^{\prime}(\lambda) d \lambda$, puis que $\theta^{\prime}(\lambda)=1$ pour presque tout $\lambda \in D_{h}$ en posant $X=$ $\left.D_{h}\right)$. Il s'ensuit que $\gamma_{h}^{\prime}(\lambda)=\left(p+p^{\prime \prime}\right)(\theta(\lambda)) u^{\prime}(\theta(\lambda))$ pour presque tout $\lambda \in D_{h}$ et la relation voulue en reportant ce résultat dans $\int_{D_{h}}\left\|\gamma_{h}^{\prime}(\lambda)\right\| d \lambda$.

Les propriétés (1)-(4) caractérisent les différences de fonctions support de corps convexes plans dans $C\left(\mathbb{S}^{1}\right)$. Notre preuve repose sur la proposition suivante :

Proposition 2. Soit $h \in \mathcal{F}$. La fonction $h$ appartient à $\mathcal{K}$ (autrement dit, $h$ est la fonction support d'un corps convexe) si et seulement si la fonction $p(\theta)=h(u(\theta))$ vérifie les conditions suivantes:

(5) $p_{\mathrm{g}}^{\prime} \leq p_{\mathrm{d}}^{\prime}$;

(6) $p+p^{\prime \prime} \geq 0$ presque partout.

Preuve. La preuve est basée sur la caractérisation suivante, due à Kallay [2] :

$$
\begin{aligned}
& \forall h \in C\left(\mathbb{S}^{1}\right), \\
& (h \in \mathcal{K}) \Leftrightarrow(\forall \theta \in I, \forall \alpha \in[0, \pi / 2], p(\theta+\alpha)+p(\theta-\alpha) \geq 2 p(\theta) \cos \alpha) .
\end{aligned}
$$

La nécessité des conditions (5) et (6) s'en déduit aisément. Inversement, les conditions (5) et (6) impliquent les relations suivantes, et par conséquent la condition de Kallay :

$$
\forall \theta \in I, \forall \alpha \in[0, \pi / 2],\left\{\begin{array}{l}
p(\theta+\alpha) \geq p(\theta) \cos \alpha+p_{\mathrm{d}}^{\prime}(\theta) \sin \alpha \\
p(\theta-\alpha) \geq p(\theta) \cos \alpha-p_{\mathrm{g}}^{\prime}(\theta) \sin \alpha .
\end{array}\right.
$$

En effet, on observe que $\left\langle\gamma_{h}^{\prime}(\lambda), u(\theta+\alpha)\right\rangle \geq 0$ presque partout sur $\left[l_{\theta}^{+}, l_{\theta+\alpha}^{+}\right]$, où $l_{\phi}^{+}=d_{h}\left(\phi, p_{\mathrm{d}}^{\prime}(\phi)\right)$ (resp. $-\left\langle\gamma_{h}^{\prime}(\lambda), u(\theta-\alpha)\right\rangle \geq 0$ presque partout sur $\left[l_{\theta-\alpha}^{-}, l_{\theta}^{-}\right]$, où $\left.l_{\phi}^{-}=d_{h}\left(\phi, p_{\mathrm{g}}^{\prime}(\phi)\right)\right)$, et la première (resp. seconde) relation s'obtient en intégrant cette inégalité sur $\left[l_{\theta}^{+}, l_{\theta+\alpha}^{+}\right]$(resp. sur $\left.\left[l_{\theta-\alpha}^{-}, l_{\theta}^{-}\right]\right)$.

ThÉORÈme 4. Les propriétés (1)-(4) caractérisent dans $C\left(\mathbb{S}^{1}\right)$ le sousespace $\mathcal{H}$ des différences de fonctions support de corps convexes de $\mathbb{E}^{2}$.

Preuve. La proposition 1 nous dit que $\mathcal{H} \subset \mathcal{F}$. Pour prouver que $\mathcal{F} \subset \mathcal{H}$, il nous suffit d'établir le résultat suivant :

$$
\forall h \in \mathcal{F}, \exists(k, r) \in \mathcal{K} \times \mathbb{R}_{+}^{*}, h+k+r \in \mathcal{K} .
$$

Ce résultat se déduit comme suit de la proposition $2:$ on commence par choisir un $r \in \mathbb{R}_{+}^{*}$ assez grand pour que l'application $\gamma_{h}$ soit $r$-lipschitzienne (cf. preuve du théorème 2 ), de sorte que la fonction $h+r$ vérifie la condition (6) compte tenu de l'expression de $\gamma_{h}^{\prime}$ (cf. preuve du théorème 3), puis on définit $k \in \mathcal{K}$ par 


$$
\begin{aligned}
& k(u(\theta))=\frac{1}{2} \sum_{\alpha \in N}\left(p_{\mathrm{g}}^{\prime}(\alpha)-p_{\mathrm{d}}^{\prime}(\alpha)\right)|\langle u(\theta), u(\alpha)\rangle|, \\
& \quad \text { où } N=\left\{\alpha \in J \mid p_{\mathrm{g}}^{\prime}(\alpha)>p_{\mathrm{d}}^{\prime}(\alpha)\right\},
\end{aligned}
$$

de sorte que la fonction $h+k+r$ vérifie la condition (5).

2. Hérissons généralisés et théorie de Brunn-Minkowski. Commençons par étendre les notions de longueur et d'aire algébriques aux hérissons généralisés :

DÉfinition. Pour tout $h \in \mathcal{H}$, appelons longueur (resp. aire) algébrique du hérisson généralisé $\mathcal{H}_{h}$ le nombre réel $l(h)$ (resp. $a(h)$ ) défini en fonction de $p(\theta)=h(u(\theta))$ par

$$
l(h)=\int_{0}^{2 \pi} p(\theta) d \theta=\int_{0}^{2 \pi}\left(p+p^{\prime \prime}\right)(\theta) d \theta+\sum_{\theta \in J}\left(p_{\mathrm{d}}^{\prime}-p_{\mathrm{g}}^{\prime}\right)(\theta)
$$

(resp.

$$
\begin{aligned}
a(h) & =\frac{1}{2} \int_{0}^{2 \pi}\left(p^{2}-\left(p^{\prime}\right)^{2}\right)(\theta) d \theta \\
& \left.=\frac{1}{2} \int_{0}^{2 \pi} p(\theta)\left(p+p^{\prime \prime}\right)(\theta) d \theta+\frac{1}{2} \sum_{\theta \in J} p(\theta)\left(p_{\mathrm{d}}^{\prime}-p_{\mathrm{g}}^{\prime}\right)(\theta)\right) .
\end{aligned}
$$

Cette définition admet implicitement deux égalités : la première se prouve en se ramenant au cas $h \in \mathcal{K}$, où il est bien connu que $L(h)=$ $\int_{0}^{2 \pi} p(\theta) d \theta$, et la seconde se déduit de la première en notant que $h^{2} \in \mathcal{H}$.

Le nombre réel $l(h)$ (resp. $a(h)$ ) s'interprète encore comme une longueur (resp. aire) algébrique et représente bien la longueur (resp. l'aire) de $\mathcal{H}_{h}$ lorsque $h$ est la fonction support d'un corps convexe de $\mathbb{E}^{2}$. En outre, $a(h)$ définit une forme quadratique sur $\mathcal{H}$, dont la forme polaire $a(f, g)$ s'interprète comme l'aire algébrique mixte des hérissons généralisés $\mathcal{H}_{f}$ et $\mathcal{H}_{g}$, et nous avons l'extension suivante de l'inégalité de Minkowski (et donc de l'inégalité isopérimétrique) :

THÉORÈme 5. Si $(f, g) \in \mathcal{H}^{2}$ est tel que $a(f)>0$, alors $a(f, g)^{2} \geq$ $a(f) a(g)$. En particulier : $\forall h \in \mathcal{H}, 4 \pi a(h) \leq l(h)^{2}$.

Preuve. Sinon le trinôme $\tau(t)=a(f+t g)=a(f)+2 t a(f, g)+t^{2} a(g)$ serait sans racine réelle et resterait donc $>0$ sur tout $\mathbb{R}$, or il existe un nombre réel $t$ tel que $l(f+t g)=0$, et donc tel que $\tau(t) \leq 0$ d'après l'inégalité de Wirtinger.

Nous terminons par un résultat qui relève du problème de Minkowski pour les hérissons généralisés (sachant que $p+p^{\prime \prime}$ s'identifie au rayon de courbure de $\mathcal{H}_{h}$ ) : 
ThÉORÈme 6 . Si $\varrho: \mathbb{R} \rightarrow \mathbb{R}$ est une application $2 \pi$-périodique qui est Lebesgue intégrable sur $I=[0,2 \pi]$ et telle que $\int_{0}^{2 \pi} \varrho(\theta) u(\theta) d \theta=0$, alors il existe un hérisson généralisé dont la fonction support $p(\theta)=h(u(\theta))$ vérifie $p+p^{\prime \prime}=\varrho$ presque partout.

Preuve. La preuve est une adaptation de celle du théorème 4 de [2]. Utilisant la caractérisation de $\mathcal{H}$, elle consiste à prouver que

$$
p(\theta)=\int_{0}^{\theta} \varrho(\alpha) \sin (\theta-\alpha) d \alpha
$$

est une fonction $2 \pi$-périodique sur $\mathbb{R}$ qui admet une dérivée absolument continue (et donc presque partout dérivable) sur $I$ et qui vérifie $p+p^{\prime \prime}=\varrho$ presque partout. Un premier calcul montre que $p$ admet une dérivée absolument continue sur $I$, à savoir

$$
p^{\prime}(\theta)=\int_{0}^{\theta} \varrho(\alpha) \cos (\theta-\alpha) d \alpha,
$$

et un second que $p+p^{\prime \prime}=\varrho$ presque partout. La condition $\int_{0}^{2 \pi} \varrho(\theta) u(\theta) d \theta=$ 0 assure quant à elle que $p$ est bien une fonction $2 \pi$-périodique sur $\mathbb{R}$.

REMARQUe. La fonction $h \in \mathcal{H}$ dont le théorème 6 nous assure l'existence n'est manifestement pas unique puisque si $h$ est la fonction support d'un polygone convexe quelconque de $\mathbb{E}^{2}$, alors la fonction $p(\theta)=h(u(\theta))$ vérifie $p+p^{\prime \prime}=0$ presque partout.

\section{Références}

[1] H. Geppert, Über den Brunn-Minkowskischen Satz, Math. Z. 42 (1937), 238-254.

[2] M. Kallay, Reconstruction of a plane convex body from the curvature of its boundary, Israel J. Math. 17 (1974), 149-161.

[3] R. Langevin, G. Levitt et H. Rosenberg, Hérissons et multihérissons, dans : Singularities, Banach Center Publ. 20, PWN, Warszawa, 1988, 245-253.

[4] Y. Martinez-Maure, De nouvelles inégalités géométriques pour les hérissons, Arch. Math. (Basel) 72 (1999), 444-453.

[5] R. Schneider, Convex Bodies: The Brunn-Minkowski Theory, Cambridge Univ. Press, 1993.

1, rue Auguste Perret

F-92500 Rueil-Malmaison

France

E-mail: martinez@esiea.fr 